\title{
Surgical repair of a giant Thoracoabdominal Aortic Aneurysm
}

\author{
Yaojun Dun ${ }^{1}$ and Xiaogang Sun $^{2}$ \\ ${ }^{1}$ Fuwai Hospital \\ ${ }^{2}$ Fuwai Hospital State Key Laboratory of Cardiovascular Disease
}

October 8, 2020

\begin{abstract}
The repair of thoracoabdominal aortic aneurysm (TAAA) require a range of surgical techniques and is associated with serious complications such as paraplegia, gastrointestinal complications and acute kidney injury. In this report, we present a case of successful giant TAAA repair. Repair was performed from proximal to distal by sequential aortic cross clamp-and-sew under normothermia in this case. To prevent visceral, renal and spinal cord ischemia, organ protection was supplemented with cerebrospinal fluid drainage, renal perfusion and selective visceral perfusion in this case.
\end{abstract}

\section{Introduction}

Thoracoabdominal aortic aneurysm (TAAA) repair remains a great challenge. Some serious complications such as paraplegia, gastrointestinal complications and acute kidney injury are still unavoidable ${ }^{1,2}$. Since the first surgical procedure of TAAA was first reported by Debakey in $1965^{3}$, several approaches have been applied to improve clinical outcome including the clamp-and-sew technique and the use of distal aortic perfusion. The maneuver of distal perfusion includes passive bypass without extracorporeal circulation and extracorporeal circulatory assistance including left heart bypass (LHB), partial cardiopulmonary bypass and hypothermic circulation arrest. Cerebrospinal fluid drainage (CSFD), renal perfusion and selective visceral perfusion (SVP) were also applied in TAAA repair to prevent visceral, renal and spinal cord ischemia ${ }^{1}$. In this report, we applied sequential aortic cross clamp-and-sew under normothermia to perform the TAAA repair, with the use of CSFD, renal perfusion and SVP. And the case in our report was also the TAAA with the largest diameter that had ever received successful surgical intervention to date. The study was approved by the institutional review board of Fuwai Hospital. A waiver of informed consent was granted due to the design of the study.

\section{Case Report}

A 53-year-old man was admitted to our hospital with thoracoabdominal fullness and discomfort after activity for about 2 years. He had no history of connective tissue disorder or aortic dissection. Computed tomography (CT) revealed a giant TAAA (Crawford extent III) with the maximal diameter of $12.7 \mathrm{~cm}$ (Figure 1a, 1b). The laboratory data were within reference range.

The operation adopted sequential aortic clamp-and-sew technique under normothermia. After induction of anesthesia, CSFD was used. The repair was performed via a standard left thoracoabdominal incision, exposing the TAAA through the sixth intercostal space and retroperitoneal space under single-lung ventilation. Heparin was given systemically.

A 24-mm tetrafurcate Dacron graft (Vascutek, Terumo, Inchinnan, Scotland) and a 10mm Dacron graft (Hemashield platinum, Maquet, Wayne, New Jersy) were used. The 10-mm graft was anastomosed to the 24-mm graft to create a five-branched graft. The new branch was anastomosed to left common iliac artery in an end-to-side fashion. Another branch was connected to the roller pump through one branch of Y-shape 
arterial cannula, and the other branch of Y-shape arterial cannula was attached to two balloon catheters (6-Fr) through a Y-connector. The roller pump received the shed blood from surgical field through left ventricle vent and right heart vent (Figure 2a).

The normal descending thoracic aorta proximal to aneurysm was cross-clamped and then transected just $2 \mathrm{~cm}$ distal to the clamp. The proximal end of $24-\mathrm{mm}$ graft and the proximal descending thoracic aorta cuff was anastomosed. Once the proximal anastomosis was completed, the lower body, visceral organs, bilateral kidneys and spinal cord could be perfused through the bypass of descending thoracic aorta to 24-mm graft to10-mm graft to abdominal aorta (Figure 2b). After 10 minutes' perfusion, aortic clamp was placed just proximal to the aortic-iliac bifurcation. The aorta is opened longitudinally from diseased segment down to the abdominal aorta. Two 6-Fr balloon catheters connected to Y-shape arterial cannula were introduced into the celiac axis and the SMA to provide SVP. Another two 6-Fr balloon catheters were introduced into bilateral renal arteries to provide cold renal perfusion with HTK fluid through a separate pump. The T10 intercostal arteries were anastomosed to the first branch of the graft with the island patch technique (Figure 2c).

Then the celiac and SMA were anastomosed to another two branches of 24-mm graft (Figure 2d). The aorta was transected just proximal to the bifurcation, leaving a cuff of abdominal aorta. The distal end of 24-mm graft and the abdominal aorta cuff was anastomosed (Figure 2e). The branch previously anastomosed to left iliac artery was re-anastomosed to right renal artery. The branch previously connected to the arterial cannula was re-anastomosed to left renal artery (Figure 2f). Thus, the reimplantation of bilateral renal arteries was completed (Figure 1c). Protamine was given to reverse the heparin after the hemostasis was completed. Then expeditious but meticulous closure was done.

The patient was extubated 22 hours after the operation. Acute kidney injury requiring dialysis and transient spinal cord injury was noted during the postoperative period. The kidney function improved gradually and the patient weaned dialysis on the 12nd postoperative day. Postoperative CT reexamination showed satisfactory results (Figure 1d). And the patient was discharged 21 days after the operation. Follow-up at 12 postoperative months showed good results.

\section{Comment}

Paraplegia, gastrointestinal complications and acute kidney injury are serious complications of TAAA repair ${ }^{1,2}$. The use of distal aortic perfusion, SVP and renal perfusion was very important to reduce these complications. And LHB was the most common maneuver to provide distal perfusion. With LHB, the bypass perfusion is started before clamping the aorta, and distal perfusion is maintained during the whole period of aortic occlusion. So it provides marginal safety during the cross-clamp period ${ }^{4}$. Our technique is different from LHB method. The downstream organs including visceral, renal and spinal cord are ischemic (about 10 minutes) during the proximal anastomosis. As soon as the anastomosis is completed, the downstream organs restore perfusion.

LHB method was performed under mild hypothermia ${ }^{4}$, while our technique was under normothermia. And LHB method can reduce proximal blood pressure and afterload to the heart during proximal anastomosis, which has the detrimental effect on the heart. But LHB circuit is very expensive, and this method carries some risks for its technical difficulty. And LHB could only provide non-pulsatile flow to viscera. The strategy of SVP in our technique was not via LHB. This strategy could directly provide pulsatile flow from heart to visceral organs through the bypass mentioned previously (Figure 2C). During SVP period, the lower body and the viscera received double perfusion from the heart and the roller pump. Interestingly, in arterial cannula connected to graft, the pulsatile flow from the heart to the viscera (flow 1) has opposite direction to the flow from the roller pump to the lower body (flow 2). Therefore, the direction of blood flow in the cannula depends on rate or pressure of two flows. If the rate of flow 1 is greater than that of flow 2 , the flow from the roller pump could only directs to the viscera, while the flow from the heart perfusates both the viscera and the lower body. If the rate of flow 1 is lesser than that of flow 2 , the lower body receives bilateral blood supply from the heart and the roller pump, while the viscera could only receive blood flow from the 
roller pump. In addition, our strategy has avoided some complicated produre related to LHB and decreased the medical expenses to great extent. Overall, it provided a safe and effective repair of giant TAAA, with satisfactory visceral organ protection.

Conflict of interest: none declared.

\section{Acknowledgements}

The authors thank Dr. Menghe Yuan for her figure drawing.

\section{References:}

1. Coselli JS, LeMaire SA, Preventza O, et al. Outcomes of 3309 thoracoabdominal aortic aneurysm repairs. J Thorac Cardiovasc Surg 2016;151:1323-37.

2. Waked K, Schepens M. State-of the-art review on the renal and visceral protection during open thoracoabdominal aortic aneurysm repair. J Vis Surg 2018;4:31.

3. DeBakey ME, Crawford ES, Garrett HE, Beall AC, Jr., Howell JF. Surgical considerations in the treatment of aneurysms of the thoraco-abdominal aorta. Ann Surg 1965;162:650-62.

4. Coselli JS, LeMaire SA. Left heart bypass reduces paraplegia rates after thoracoabdominal aortic aneurysm repair. Ann Thorac Surg 1999;67:1931-4; discussion 53-8.

\section{Figure Legends}

Figure 1. (a)(b) Preoperative CT scan; (c) Completion of TAAA repair; (d) Postoperative CT scan (P: proximal, D: distal, 1: T10 intercostal arteries, 2: superior mesenteric artery, 3, celiac axis, 4: left renal artery, 5: right renal artery).

Figure 2. (a) One branch was connected to left iliac artery and another branch was connected to pump; (b) The proximal end of graft was anastomosed with descending aorta; (c) Two balloon catheters were introduced into SMA and celiac to provide SVP and the other two catheters were inserted into bilateral renal arteries to provide cold crystalloid perfusion. (d) Three branches were anastomosed with T10 intercostal arteries, SMA and the celiac, respectively; (e) The distal end of main graft was anastomosed with abdominal aorta cuff; (f) The remaining two branches were re-anastomosed with bilateral renal arteries. (SMA: superior mesenteric artery, LRA: left renal artery, RRA: right renal artery)
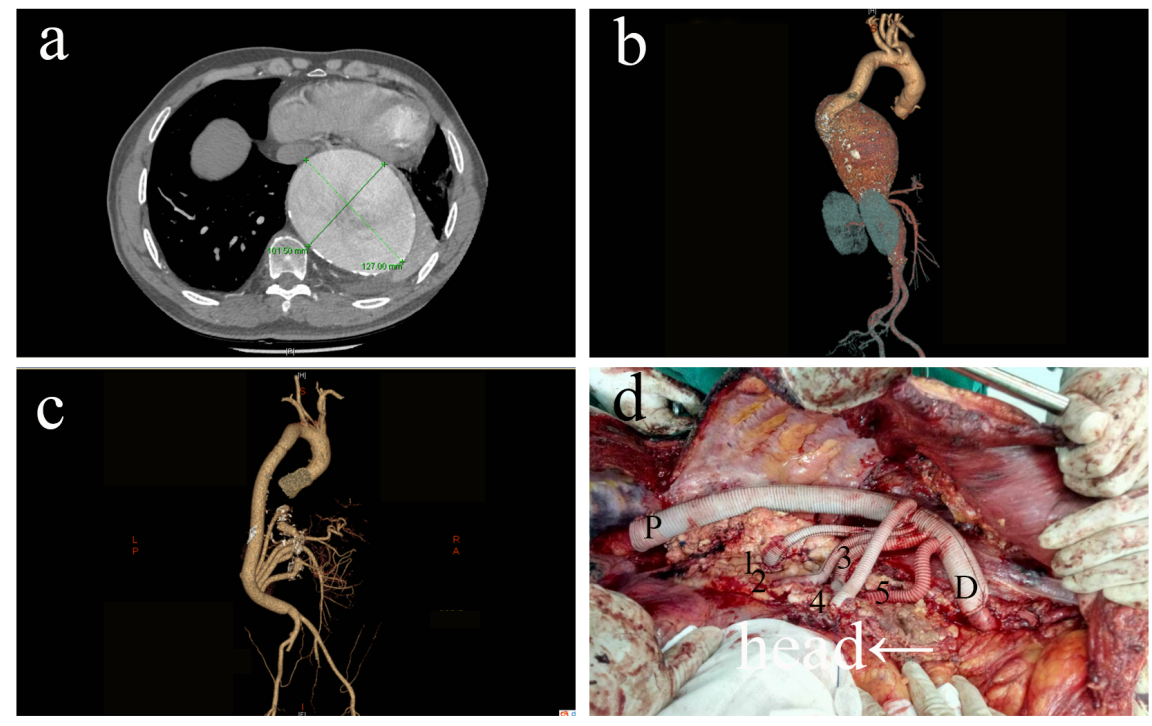


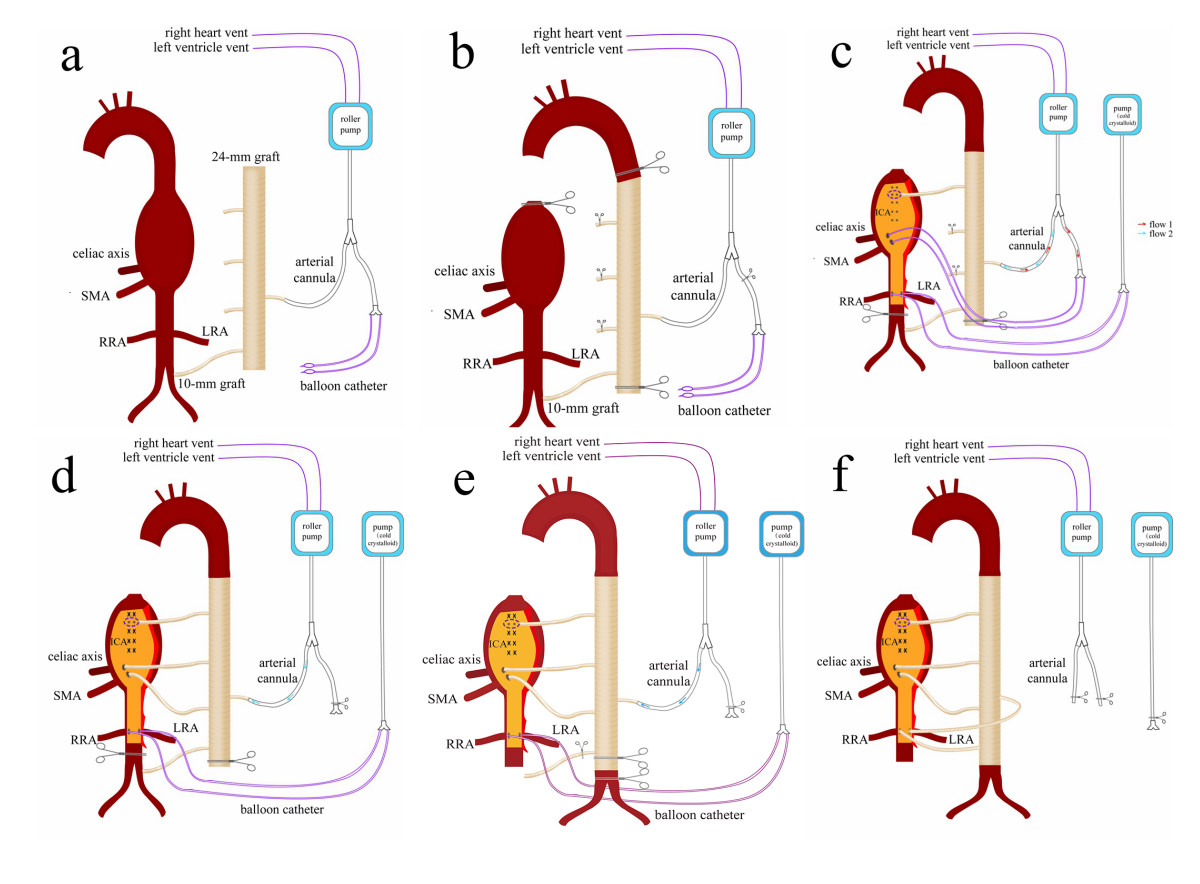

